\title{
Bases juridiques pour le quotidien du médecin
}

\author{
Anne-Sylvie Thiébaud Noria, Michelle Salathéb \\ ${ }^{a}$ avocate, division Service juridique de la FMH, cheffe de projet; ${ }^{b}$ lic. iur., MAE, secrétaire générale adjointe de I'ASSM
}

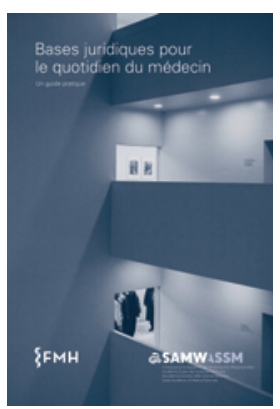

De nombreuses dispositions légales et réglementaires accompagnent le médecin dans son activité quotidienne et comme non-juriste, il lui est souvent difficile de garder une vue d'ensemble et de connaître les règles qui s'appliquent à son travail. En publiant le guide pratique "Bases juridiques pour le quotidien du médecin», l'Académie Suisse des Sciences Médicales (ASSM) et la FMH espèrent offrir un instrument utile aux praticiens. La troisième édition de ce guide répond de manière simple et pratique à diverses questions juridiques fréquemment posées. Sa nouvelle structure tient compte des changements législatifs et politiques intervenus depuis sa dernière parution en 2013. Les dispositions légales tout comme la jurisprudence ont été mises à jour jusqu'au milieu de l'année 2019. Le guide pratique a été rédigé par une équipe composée de collaborateurs de la FMH, du Service juridique de la FMH, de l'ASSM, de l'ISFM et de plusieurs auteurs externes. Un conseil scientifique a accompagné sa rédaction.

\section{Concentration sur les aspects relevant de la pratique}

Les questions juridiques les plus fréquentes et les plus importantes pouvant se poser dans la pratique quotidienne des cabinets médicaux et des hôpitaux sont exposées sur 200 pages. Les textes sont courts, à dessein, et ont pour but de donner les premiers renseignements sur les thématiques abordées. Aux personnes intéressées qui souhaiteraient approfondir les différents sujets, consulter un lien ou une disposition légale, le guide propose plus de 600 notes de bas de page. Le document est disponible en allemand et en français.

\section{Accents thématiques}

- Le chapitre 1 présente les bases, la fonction du droit dans la vie quotidienne du médecin et la hiérarchie des normes, de la Constitution fédérale aux réglementations de l'ASSM et de la FMH.

- Le chapitre 2 donne un aperçu des conditions générales régissant l'activité médicale. Il passe en revue la formation prégraduée, postgraduée et continue, les conditions à l'exercice de la médecine et à l'ad- mission à facturer à la charge des caisses, les devoirs professionnels et la réglementation des assurances sociales et privées.

- Le chapitre 3 aborde les bases encadrant la pratique médicale: il s'agit par exemple du contrat de soins, de l'information au patient et du consentement de ce dernier, de la tenue du dossier médical, mais également du dossier électronique du patient, de l'utilisation de médicaments, de produits stupéfiants et de dispositifs médicaux. Il expose par ailleurs les dispositions principales concernant le traitement de patients contenues dans la LAMal, la LAA, la LAM et la LAI.

- Le chapitre 4 se concentre sur les situations particulières se présentant dans le cadre d'un traitement, de l'analyse génétique à la médecine reproductive, en passant par l'interruption volontaire de grossesse, mais aussi les mesures forcées ou l'aide au suicide.

- Le chapitre 5 aborde le thème de la recherche et des registres, ainsi que les droits et les devoirs qui en découlent.

- Le chapitre 6 est consacré au secret professionnel, à la protection des données, à la rédaction de rapports et à la facturation.

- Le chapitre 7 décrit le cadre et les dispositions légales s'agissant des expertises médicales.

- Le chapitre 8 aborde la question de la responsabilité du médecin: du droit du patient à consulter son dossier et à en effectuer des copies, à la responsabilité civile et pénale du médecin.

- Le chapitre 9 a pour vocation de donner des repères au praticien en tant qu'employé ou employeur, de l'acceptation d'un poste dans un hôpital à l'ouverture d'un cabinet, en passant par tous les devoirs que cela implique, comme par exemple les dispositions régissant la protection de la santé en cas de maternité des assistantes médicales.

Le guide pratique comporte encore une liste des auteurs des trois éditions et des membres du conseil scientifique, ainsi qu'un index détaillé des mots clés. Il peut être téléchargé à l'adresse www.fmh.ch $\rightarrow$ Prestations $\rightarrow$ Droit ou assm.ch/guides-pratiques; une version imprimée peut également être commandée gratuitement à cette dernière adresse. 